Objectives: We sought to quantify rates of newly treated diabetes mellitus, hypertension, and venous thromboembolism (VTE) associated with oral glucocorticoid exposure in children. We hypothesized that oral glucocorticoids were associated with each complication in a dose-dependent manner, most strongly with current or recent glucocorticoid exposure.

Methods: Using Medicaid claims data (2000-2010), we identified children ages 1-18 diagnosed with immune-mediated diseases (inflammatory bowel disease, juvenile idiopathic arthritis, or psoriasis) or a non-immune comparator condition (attention-deficit/hyperactivity disorder) based on diagnostic codes \pm pharmacy claims. We studied time-varying oral glucocorticoid exposures using pharmacy claims after a $>6$-month glucocorticoid-free baseline period, categorizing dose as low $(<0.25 \mathrm{mg} / \mathrm{kg} /$ day $)$, medium $(0.25-0.99 \mathrm{mg} / \mathrm{kg} / \mathrm{day})$, or high $(\geq 1 \mathrm{mg} / \mathrm{kg} / \mathrm{day})$ based on prescribed prednisone-equivalent dosage and age-/sex-imputed weights. Primary outcomes were incident treatment of diabetes (type 1 or 2), hypertension, and VTE. We used Cox regression and weighted cumulative exposure models ${ }^{1}$ to estimate adjusted hazard ratios (aHRs) and number needed to harm (NNH) for varying patterns of glucocorticoid exposure.

Results: We followed 932,517 children (21.3\% glucocorticoid-exposed) for 1.6 million person-years. Glucocorticoid usage varied by disease (Table). After adjusting for age, sex, race/ethnicity, calendar year, inclusion diagnosis, comorbidities, other medications, and healthcare usage, we found strong dose- and time-dependent relationships between glucocorticoid exposure and rates of newly treated diabetes, hypertension, and VTE (Figure 1). These effects increased with longer durations of exposure and declined within 6 months after stopping (Figure 2). Sustained low-dose exposures (e.g., $0.1 \mathrm{mg} / \mathrm{kg} /$ day) appeared relatively safe $(\mathrm{aHR}<1.2)$, but risks increased even with brief high-dose exposures $(2 \mathrm{mg} / \mathrm{kg} /$ day $x 7$ days) (aHR 1.7-2.2). Risk differences were highest for hypertension (number needed to harm [NNH]: $2 \mathrm{mg} / \mathrm{kg} /$ day $x 7$ days, 6,206-17,894; $2 \mathrm{mg} /$ $\mathrm{kg} /$ day $\times 30$ days, 233-671) and lowest for VTE (NNH: $2 \mathrm{mg} / \mathrm{kg} /$ day $\times 7$ days, $54,886-612,350 ; 2 \mathrm{mg} / \mathrm{kg} /$ day $\times 30$ days, 1,977-22,045). Absolute risks were higher for children with immune-mediated diseases (e.g., diabetes, $2 \mathrm{mg} / \mathrm{kg} /$ day $\times 30$ days, NNH: 424-691) than for those with ADHD (NNH: 959).

Table. Distribution of dose and duration of oral glucocorticoids by disease cohort.

\begin{tabular}{lcccc}
\hline Cohort & Aspect & $\begin{array}{c}25 \text { th } \\
\%\end{array}$ & $\begin{array}{c}50 \text { th } \\
\%\end{array}$ & $\begin{array}{c}75 \text { th } \\
\%\end{array}$ \\
\hline ADHD & Duration & 5 & 5 & 7 \\
ADHD & Dose & 0.41 & 0.74 & 1.24 \\
IBD & Duration & 5 & 27 & 47 \\
IBD & Dose & 0.29 & 0.59 & 0.92 \\
JIA & Duration & 5 & 9 & 30 \\
JIA & Dose & 0.16 & 0.33 & 0.88 \\
PSO & Duration & 5 & 6 & 10 \\
PSO & Dose & 0.3 & 0.63 & 1.14 \\
\hline
\end{tabular}

Units: duration, days; dose, $\mathrm{mg} / \mathrm{kg} /$ day.
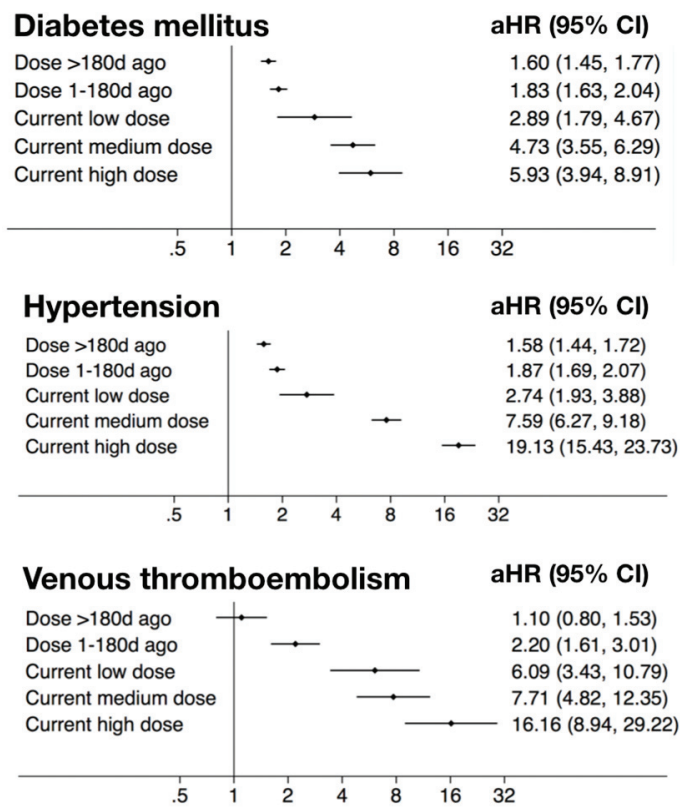

Figure 1. Associations of glucocorticoid timing and dose with newly treated outcomes based on Cox regression models.
aHR, adjusted hazard ratio; $\mathrm{Cl}$, confidence interval
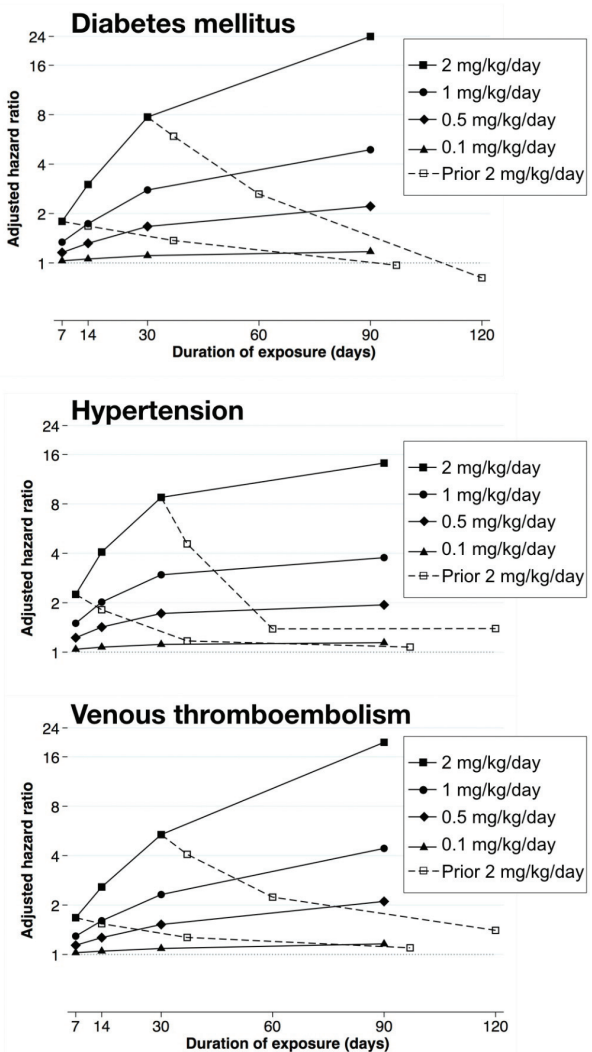

Figure 2. Associations of glucocorticoid timing and dose with newly treated outcomes based on weighted cumulative exposure models.

Line plots show associations of daily glucocorticoid dose, duration, and recency with outcomes.

Conclusion: In children with various chronic conditions, current oral glucocorticoid use is strongly associated with newly treated diabetes, hypertension, and VTE in a dose- and duration-dependent fashion. Hypertension is a more common glucocorticoid-related complication than diabetes or VTE, but in absolute terms, all of these complications are uncommon in children.

REFERENCES:

[1] Sylvestre MP, Abrahamowicz M. Stat Med. 2009;28(27):3437-3453.

Disclosure of Interests: Daniel Horton Grant/research support from: Bris tol-Myers Squibb, for unrelated research, Fenglong Xie: None declared, Lang Chen: None declared, Melissa L. Mannion: None declared, Jeffrey Curtis: None declared, Brian Strom: None declared, Timothy Beukelman Consultant for: Novartis, UCB

DOI: 10.1136/annrheumdis-2019-eular.1622

\section{FRI0535 COMPARISON OF SERUM BIOMARKERS FOR THE DIAGNOSIS OF MACROPHAGE ACTIVATION SYNDROME COMPLICATING SYSTEMIC JUVENILE IDIOPATHIC ARTHRITIS: CLINICAL SIGNIFICANCE OF SERUM NEOPTERIN LEVELS}

Hitoshi Irabu $^{1}$, Masaki Shimizu ${ }^{1}$, Maiko Takakuwa ${ }^{1}$, Naoto Sakumura ${ }^{1}$, Natsumi Inoue ${ }^{1}$, Mao Mizuta ${ }^{1}$, Yasuo Nakagishi ${ }^{2}$, Akihiro Yachie ${ }^{1} .{ }^{1}$ Graduate School of Medical Sciences, Kanazawa University, Department of Pediatrics, Kanazawa, Japan; ${ }^{2}$ Hyogo Prefectural Kobe Children's Hospital, Department of Pediatric Rheumatology, Kobe, Japan

Background: Macrophage activation syndrome (MAS) is a severe lifethreatening condition that complicates systemic juvenile idiopathic arthritis (s-JIA). Differentiating MAS from these conditions is essential for selecting appropriate therapeutic interventions in a timely manner. However, there is no definite biomarkers that can effectively diagnose MAS. 
Objectives: Our study aimed to compare the accuracy of serum biomarkers for the diagnosis of MAS complicating S-JIA and to investigate the clinical significance of serum neopterin levels as an indicator of disease activity and diagnosis of MAS complicating S-JIA.

Methods: Serum cytokine levels (neopterin, IL-18, and CXCL9 and soluble tumor necrosis factor receptor type I (sTNFR-I) and II were determined by enzyme-linked immunosorbent assay in 78 patients with S-JIA, including 21 with MAS. The accuracy of these levels for the diagnosis of MAS were compared. Next, serum neopterin levels, in total 125 patients with S-JIA, including 30 with MAS, 15 with Epstein-Barr virus-induced hemophagocytic lymphohistiocytosis (EBV-HLH), and 15 with Kawasaki disease (KD), as well as 28 healthy controls (HCs) were analysed. Results were compared with the clinical features of MAS.

Results: Receiver operating characteristic curve analysis revealed area under the curve values and cut off values of neopterin, IL-18, CXCL9, sTNFR-II/I ratio and ferritin were $0.9465 / 19.5 \mathrm{nmol} / \mathrm{l}, 0.8895 / 69250 \mathrm{ng} / \mathrm{ml}$, $0.9333 / 3130 \mathrm{pg} / \mathrm{ml}, 0.9395 / 3.796$ and $0.8671 / 2560 \mathrm{ng} / \mathrm{ml}$, respectively. Serum neopterin levels were significantly elevated in patients with MAS and EBV-HLH compared with those in patients with acute-phase S-JIA and KD. Serum neopterin levels profoundly and rapidly increased as MAS developed and correlated positively with disease activity.

Conclusion: Serum neopterin levels may be used as a promising indicator of disease activity in S-JIA and MAS and for evaluating it. It may also be a useful marker to diagnose the transition to MAS from activephase s-JIA.

\section{REFERENCES:}

[1] Huber C, et al. J Exp Med 1984;160:310-6.

[2] Bracaglia C, et al. Ann Rheum Dis 2017;76:166-72.

[3] Shimizu M, et al. Clin Exp Immunol 2018;191:349-355.

Disclosure of Interests: None declared

DOI: 10.1136/annrheumdis-2019-eular.5537

\section{FRI0536 FAMILIAL MEDITERRANEAN FEVER (FMF): A SINGLE CENTEREXPERIENCE FROM TURKEY}

Ayșe Tanatar, Şerife Gül Karadağ, Hafize Emine Sonmez, Mustafa Çakan, Nuray Aktay Ayaz. University of Health Science, Kanuni Sultan Süleyman Research and Training Hospital, Pediatric Rheumatology, Istanbul, Turkey

Background: Familial Mediterranean fever (FMF) is the most common monogenic autoinflammatory disease mainly affecting ethnic groups living at Mediterranean region. Since the discovery of the Mediterranean FeVer (MEFV) gene, molecular genetic testing has been used as a diagnostic adjunct especially in atypical cases $(1,2)$. Although substantial progress had been achieved about the etiopathogenetic mechanisms of FMF during the past 20 years, the diagnosis is still based on clinical criteria.

Objectives: To define the demographic, clinical and laboratory characteristics of children with FMF and then to compare the identification capacity of 3 validated FMF diagnostic criteria (Tel-Hashomer, Livneh and Pediatric) at our cohort (3-5).

Methods: The medical records of 1685 children diagnosed and followed up as FMF were reviewed retrospectively. All patients were evaluated for three diagnostic criteria.

Results: A total of 1685 children (839 girls, 846 boys) were involved to the study. Family history of FMF was positive in $46.1 \%$. The mean sstandard deviation of current age, age at symptom onset, age at diag nosis were $13 \pm 5.4,5.4 \pm 4.05,7.9 \pm 4.1$ years, respectively. Median (min max) follow-up period was $3(0.5-18)$ years. Among 1685 patients, $82.8 \%$ had fever, $78.2 \%$ had abdominal pain, $36.1 \%$ had arthritis, $22.6 \%$ had chest pain and $16.6 \%$ had erysipelas-like erythema. Three patients had biopsy proven amyloidosis. Concomitant disease was present in 140 (8.3\%) patients. Most of them (40.7\%) were diagnosed with juvenile idiopathic arthritis and FMF. Henoch-Schönlein vasculitis was observed in 35 (25\%) patients. Median (min-max) PRAS score was 7 (3-13). Forty-four patients $(2.6 \%)$ were unresponsive to adequate doses of colchicine. Among them, 16 (36.4\%) were treated with anakinra and 28 (63.6\%) received canakinumab. Children homozygous for $M 694 \mathrm{~V}$ were found to have more severe course of disease and higher PRAS scores $(p<0.001)$. Furthermore, $34(77.3 \%)$ of colchicine resistant patients carried at least one $M 694 \mathrm{~V}$ variant. When we applied the diagnostic criteria to our cohort, $99.5 \%$ met the Livneh criteria, $91.6 \%$ fulfilled the pediatric criteria and $82.9 \%$ satisfied the Tel-Hashomer criteria.

Conclusion: This is the largest pediatric cohort studied and presented since now. We believe that the large number of our cohort is convincing at the point of discussing phenotype-genotype relations. We confirmed that carrying $M 694 \mathrm{~V}$ mutation is associated with increased disease severity. On the other hand, we compared two adult and one pediatric validated diagnostic criteria at a largest group of children with FMF.

\section{REFERENCES}

[1] Ancient missense mutations in a new member of the RoRet gene family are likely to cause familial Mediterranean fever. The International FMF Consortium (1997). Cell 90:797-807

[2] A candidate gene for familial Mediterranean fever (1997). Nat Genet 17:25-31.

[3] Sohar E, Gafni J, Pras M, Heller H (1967) Familial Mediterranean fever. A survey of 470 cases and review of the literature. The American journal of medicine 43:227-253

[4] Livneh A, Langevitz P, Zemer D, Zaks N, Kees S, Lidar T, Migdal A, Padeh S, Pras M (1997) Criteria for the diagnosis of familial Mediterranean fever. Arthritis and rheumatism 40:1879-1885.

[5] Yalcinkaya F, Ozen S, Ozcakar ZB, Aktay N, Cakar N, Duzova A, Kasapcopur O, Elhan AH, Doganay B, Ekim M, Kara N, Uncu N, Bakkaloglu A (2009) A new set of criteria for the diagnosis of familial Mediterranean fever in childhood. Rheumatology (Oxford, England) 48:395-398.

Disclosure of Interests: None declared

DOI: 10.1136/annrheumdis-2019-eular.6558

\section{FRI0537 LONG-TERM OUTCOMES AND TREATMENT EFFICACY IN PATIENTS WITH TNF RECEPTOR-ASSOCIATED AUTOINFLAMMATORY SYNDROME (TRAPS): A SERIES OF 290 CASES FROM THE EUROFEVER/EUROTRAPS INTERNATIONAL REGISTRY}

Riccardo Papa ${ }^{1}$, Thirusha Lane ${ }^{2}$, Taryn Youngstein ${ }^{2}$, Tamer Rezk $^{2}$, Charalampia Papadopoulou ${ }^{3}$, Nicolino Ruperto ${ }^{1}$, Paul Brogan ${ }^{3}$, Philip N. Hawkins ${ }^{2}$ Patricia Woo ${ }^{3}$, Marco Gattorno ${ }^{1}$, Helen J. Lachmann ${ }^{2} .{ }^{1}$ IRCCS Istituto Giannina Gaslini, Clinica Pediatrica e Reumatologia, Genova, Italy; ${ }^{2}$ Division of Medicine, Royal Free Campus, University College London, National Amyloidosis Centre, LONDON, United Kingdom; ${ }^{3}$ UCL Great Ormond Street Institute of Child Health, Department of Infection, Inflammation and Rheumatology, LONDON, United Kingdom

Background: Tumour necrosis factor (TNF) receptor-associated periodic syndrome (TRAPS) is one of the best known monogenic auto-inflammatory disorder resulting from an autosomal dominant variation in the TNF super family receptor $1 \mathrm{~A}$ (TNFRSF1A) gene.

Objectives: To define best treatment approach in patients with TRAPS and effect on long-term outcomes.

Methods: We reviewed all data on patients with TNFRSF1A variants enrolled in the Eurofever/EUROTRAPS international registry.

Results: Data on 290 patients were available. Patients with R92Q, P46L or intronic variants (49\%) displayed milder disease than 147 patients with mutations affecting other coding regions, with less frequent abdominal pain and skin rashes $(P<0.01)$, higher efficacy rate of colchicine as maintenance treatment, and none developed AA amyloidosis. Almost $90 \%$ of patients with exon mutations required maintenance therapy. Anti-interleukin (IL) $1 \beta$ drugs were the most frequently used (47 patients), with the highest efficacy rate (>90\% complete response), while Etanercept was less effectively used and discontinued in $72 \%$ of patients. No patients on antiIL1 $\beta$ treatment developed amyloidosis and 10 patients with amyloidosis have been successfully treated with anti IL-1 agents with preservation of native renal function in 7 and excellent long-term transplant function in 2. Nine women had a history of failure to conceive and seven had successful pregnancies without fertility treatment following complete disease control with anti-IL1 $\beta$ drugs. Long term safety profiles for anti IL-1 agents were excellent even in the presence of comorbidity.

Conclusion: Anti-IL1 $\beta$ drugs are the best maintenance treatment in TRAPS with potential to reverse the most serious disease complications of AA amyloidosis and infertility. The diagnosis of TRAPS should be considered very carefully in patients carrying $\mathrm{R} 92 \mathrm{Q}, \mathrm{P} 46 \mathrm{~L}$ or intronic TNFRSF1A variants.

\section{REFERENCES:}

[1] Lachmann HJ, Papa R, Gerhold K, Obici L, Touitou I, Cantarini L, et al. The phenotype of TNF receptor-associated autoinflammatory syndrome (TRAPS) at presentation: a series of 158 cases from the Eurofever/EUROTRAPS international registry. Annals of the rheumatic diseases. 2014;73 (12):2160-7.

[2] Papa R, Doglio M, Lachmann HJ, Ozen S, Frenkel J, Simon A, et al. A web-based collection of genotype-phenotype associations in hereditary 